\title{
Timed Automata with Asynchronous Processes: Schedulability and Decidability
}

\author{
Elena Fersman, Paul Pettersson, and Wang $\mathrm{Yi}^{\star}$
}

Uppsala University, Sweden

\begin{abstract}
In this paper, we exend timed automata with asynchronous processes i.e. tasks triggered by events as a model for real-time systems. The model is expressive enough to describe concurrency and synchronization, and real time tasks which may be periodic, sporadic, preemptive or non-preemptive. We generalize the classic notion of schedulability to timed automata. An automaton is schedulable if there exists a scheduling strategy such that all possible sequences of events accepted by the automaton are schedulable in the sense that all associated tasks can be computed within their deadlines. We believe that the model may serve as a bridge between scheduling theory and automata-theoretic approaches to system modeling and analysis. Our main result is that the schedulability checking problem is decidable. To our knowledge, this is the first general decidability result on dense-time models for real time scheduling without assuming that preemptions occur only at integer time points. The proof is based on a decidable class of updatable automata: timed automata with subtraction in which clocks may be updated by subtractions within a bounded zone. The crucial observation is that the schedulability checking problem can be encoded as a reachability problem for such automata. Based on the proof, we have developed a symbolic technique and a prototype tool for schedulability analysis.
\end{abstract}

\section{Introduction}

One of the most important issues in developing real time systems is schedulability analysis prior to implementation. In the area of real time scheduling, there are well-studied methods [8] e.g. rate monotonic scheduling, that are widely applied in the analysis of periodic tasks with deterministic behaviours. For non-periodic tasks with non-deterministic behaviours, there are no satisfactory solutions. There are approximative methods with pessimistic analysis e.g. using periodic tasks to model sporadic tasks when control structures of tasks are not considered. The advantage with automata-theoretic approches e.g. using timed automata in modeling systems is that one may specify general timing constraints on events and model other behavioural aspects such as concurrency and synchronization. However, it is not clear how timed automata can be used

\footnotetext{
* Corresponding author: Wang Yi, Department of Information Technology, Uppsala University, Box 325, 751 05, Uppsala, Sweden. yi@docs.uu.se
} 
for schedulability analysis because there is no support for specifying resource requirements and hard time constraints on computations e.g. deadlines.

Following the work of [11], we study an extended version of timed automata with asynchronous processes i.e. tasks triggered by events. A task is an executable program characterized by its worst case execution time and deadline, and possibly other parameters such as priorities etc for scheduling. The main idea is to associate each location of an automaton with a task (or a set of tasks in the general case). Intuitively a transition leading to a location in the automaton denotes an event triggering the task and the guard (clock constraints) on the transition specifies the possible arrival times of the event. Semantically, an automaton may perform two types of transitions. Delay transitions correspond to the execution of running tasks (with highest priority) and idling for the other waiting tasks. Discrete transitions correspond to the arrival of new task instances. Whenever a task is triggered, it will be put in the scheduling queue for execution (i.e. the ready queue in operating systems). We assume that the tasks will be executed according to a given scheduling strategy e.g. FPS (fixed priority scheduling) or EDF (earliest deadline first). Thus during the execution of an automaton, there may be a number of processes (released tasks) running in parallel (logically).

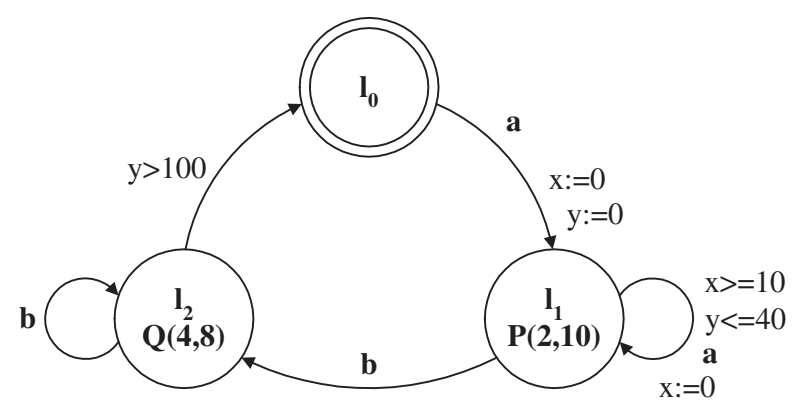

Fig. 1. Timed automaton with asynchronous processes.

For example, consider the automaton shown in Figure1 It has three locations $l_{0}, l_{1}, l_{2}$, and two tasks $P$ and $Q$ (triggered by $a$ and $b$ ) with computing time and relative deadline in brackets $(2,10)$, and $(4,8)$ respectively. The automaton models a system starting in $l_{0}$ may move to $l_{1}$ by event $a$ at any time, which triggers the task $P$. In $l_{1}$, as long as the constraints $x \geq 10$ and $y \leq 40$ hold and event $a$ occurs, a copy of task $P$ will be created and put in the scheduling queue. However, in $l_{1}$, it can not create more than 5 instances of $P$ because the constraint $y \leq 40$ will be violated after 40 time units. In fact, every copy will be computed before the next instance arrives and the scheduling queue may contain at most one task instance and no task instance will miss its deadline in $l_{1}$. In $l_{1}$, the system is also able to accept $b$, trigger $Q$ and then switch to $l_{2}$. In $l_{2}$, because there is no constraints labelled on the $b$-transition, it may accept 
any number of b's, and create any number of $Q$ 's in 0 time. This is the so-called zeno-behavior. However, after more than two copies of $Q$, the queue will be non-schedulable. This means that the system is non-schedulable. Thus, zenobehaviour will correspond to non-schedulability, which is a natural property of the model.

We shall formalize the notion of schedulability in terms of reachable states. A state of an extended automaton will be a triple $(l, u, q)$ consisting of a location $l$, a clock assignment $u$ and a task queue $q$. The task queue contains pairs of remaining computing times and relative deadlines for all released tasks. Naturally, a state $(l, u, q)$ is schedulable if $q$ is schedulable in the sense there exists a scheduling strategy with which all tasks in $q$ can be computed within their deadlines. An automaton is schedulable if all reachable states of the automaton are schedulable. Note that the notion of schedulability here is relative to the scheduling strategy. A task queue which is not schedulable with one scheduling strategy, may be schedulable with another strategy. In [11], we have shown that under the assumption that the tasks are non-preemptive, the schedulability checking problem can be transformed to a reachability problem for ordinary timed automata and thus it is decidable. The result essentially means that given an automaton it is possible to check whether the automaton is schedulable with any non-preemptive scheduling strategy. For preemptive scheduling strategies, it has been suspected that the schedulability checking problem is undecidable because in preemptive scheduling we must use stop-watches to accumulate computing times for tasks. It appears that the computation model behind preemptive scheduling is stop-watch automata for which it is known that the reachability problem is undecidable. Surprisingly the above intuition is wrong. In this paper, we establish that the schedulability checking problem for extended timed automata is decidable for preemptive scheduling. In fact, our result applies to not only preemptive scheduling, but any scheduling strategy. That is, for a given extended timed automata, it is checkable if there exists a scheduling strategy (preemtive or non-preemtive) with which the automaton is schedulable. The crucial observation in the proof is that the schedulability checking problem can be translated to a reachability problem for a decidable class of updatable automata, that is, timed automata with subtraction where clocks may be updated with subtraction only in a bounded zone.

The rest of this paper is organized as follows: Section 2 presents the syntax and semantics of timed automata extended with tasks. Section 3 describes scheduling problems related to the model. Section 4 is devoted to the main proof that the schedulability checking problem for preemptive scheduling is decidable. Section 5 concludes the paper with summarized results and future work, as well as a brief summary and comparison with related work.

\section{Timed Automata with Tasks}

Let $\mathcal{P}$, ranged over by $P, Q, R$, denote a finite set of task types. A task type may have different instances that are copies of the same program with different inputs. We further assume that the worst case execution times and hard deadlines of 
tasks in $\mathcal{P}$ are known 1 . Thus, each task $P$ is characterized as a pair of natural numbers denoted $P(C, D)$ with $C \leq D$, where $C$ is the worst case execution time of $P$ and $D$ is the relative deadline for $P$. We shall use $C(P)$ and $D(P)$ to denote the worst case execution time and relative deadline of $P$ respectively.

As in timed automata, assume a finite alphabet $\mathcal{A} c t$ ranged over by $a, b$ etc and a finite set of real-valued clocks $\mathcal{C}$ ranged over by $x_{1}, x_{2}$ etc. We use $\mathcal{B}(\mathcal{C})$ ranged over by $g$ to denote the set of conjunctive formulas of atomic constraints in the form: $x_{i} \sim C$ or $x_{i}-x_{j} \sim D$ where $x_{i}, x_{j} \in \mathcal{C}$ are clocks, $\sim \in\{\leq,<, \geq,>\}$, and $C, D$ are natural numbers. The elements of $\mathcal{B}(\mathcal{C})$ are called clock constraints.

Definition 1. A timed automaton extended with tasks, over actions $\mathcal{A}$ ct, clocks $\mathcal{C}$ and tasks $\mathcal{P}$ is a tuple $\left\langle N, l_{0}, E, I, M\right\rangle$ where

- $\left\langle N, l_{0}, E, I\right\rangle$ is a timed automaton where

- $N$ is a finite set of locations ranged over by $l, m, n$,

- $l_{0} \in N$ is the initial location, and

- $E \subseteq N \times \mathcal{B}(\mathcal{C}) \times \mathcal{A} c t \times 2^{\mathcal{C}} \times N$ is the set of edges.

- $I: N \mapsto \mathcal{B}(\mathcal{C})$ is a function assigning each location with a clock constraint (a location invariant).

$-M: N \hookrightarrow \mathcal{P}$ is a partial function assigning locations with task 2 .

Intuitively, a discrete transition in an automaton denotes an event triggering a task annotated in the target location, and the guard on the transition specifies all the possible arrival times of the event (or the annotated task). Whenever a task is triggered, it will be put in the scheduling (or task) queue for execution (corresponding to the ready queue in operating systems).

Clearly extended timed automata are at least as expressive as timed automata; in particular, if $M$ is the empty mapping, we will have ordinary timed automata. It is a rather general and expressive model. For example, it may model time-triggered periodic tasks as a simple automaton as shown in Figure 2(a) where $P$ is a periodic task with computing time 2, deadline 8 and period 20. More generally it may model systems containing both periodic and sporadic tasks as shown in Figure 2(b) which is a system consisting of 4 tasks as annotation on locations, where $P_{1}$ and $P_{2}$ are periodic with periods 10 and 20 respectively (specified by the constraints: $\mathrm{x}=10$ and $\mathrm{x}=20$ ), and $Q_{1}$ and $Q_{2}$ are sporadic or event driven by event $a$ and $b$ respectively.

In general, there may be a number of released tasks running logically in parallel. For example, an instance of $Q_{2}$ may be released before the preceding instance of $P_{1}$ is finished because there is no constraint on the arrival time of $b_{2}$. This means that the queue may contains at least $P_{1}$ and $Q_{2}$. In fact, instances of all four task types may appear in the queue at the same time.

\footnotetext{
1 Tasks may have other parameters such as fixed priority for scheduling and other resource requirements e.g. on memory consumption. For simplicity, in this paper, we only consider computing time and deadline.

${ }^{2}$ Note that $M$ is a partial function meaning that some of the locations may have no task. Note also that we may also associate a location with a set of tasks instead of a single one. It will not introduce technical difficulties.
} 
Shared Variables. To have a more general model, we may introduce data variables shared among automata and tasks. For example, shared variables can be used to model precedence relations and synchronization between tasks. Note that the sharing will not add technical difficulties as long as their domiains are finite. For simplicity, we will not consider sharing in this paper. The only requirement on the completion of a task is given by the deadline. The time when a task is finished does not effect the control behavior specified in the automaton.

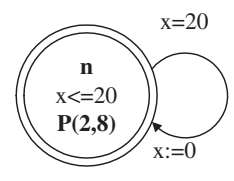

(a)

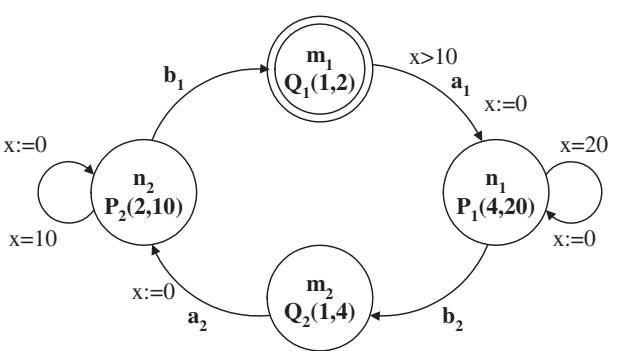

(b)

Fig. 2. Modeling Periodic and Sporadic Tasks.

Parallel Composition. To handle concurrency and synchronization, a parallel composition of extended timed automata may be defined as a product automaton in the same way as for ordinary timed automata (e.g. see [16]). Note that the parallel composition here is only an operator to construct models of systems based on their components. It has nothing to do with multi-processor scheduling. A product automaton may be scheduled to run on a one- or multi-processor system.

Semantically, an automaton may perform two types of transitions. Delay transitions correspond to the execution of running tasks with highest priority (or earliest deadline) and idling for the other tasks waiting to run. Discrete transitions corresponds to the arrivals of new task instances.

We represent the values of clocks as functions (i.e. clock assignments) from $\mathcal{C}$ to the non-negative reals $\mathcal{R}_{\geq 0}$. We denote by $\mathcal{V}$ the set of clock assignments for $\mathcal{C}$. Naturally, a semantic state of an automaton is a triple $(l, u, q)$ where $l$ is the current location, $u \in \mathcal{V}$ denotes the current values of clocks, and $q$ is the current task queue. We assume that the task queue takes the form: $\left[P_{1}\left(c_{0}, d_{0}\right), P_{2}\left(c_{1}, d_{1}\right) \ldots P_{n}\left(c_{n}, d_{n}\right)\right]$ where $P_{i}\left(c_{i}, d_{i}\right)$ denotes a released instance of task type $P_{i}$ with remaining computing time $c_{i}$ and relative deadline $d_{i}$.

Assume that there are a fixed number of processors running the released task instances according to a certain scheduling strategy Sch e.g. FPS (fixed priority scheduling) or EDF (earliest deadline first) which sorts the task queue whenever new tasks arrives according to task parameters e.g. deadlines. An action transition will result in a sorted queue including the newly released tasks by the 
transition. A delay transition with $t$ time units is to execute the task in the first positon of the queue with $t$ time units. Thus the delay transition will decrease the computing time of the first task with $t$. If its computation time becomes 0 , the task should be removed from the queue. Moreover, all deadlines in the queue will be decreased by $t$ (time has progressed by t). To summarize the above intuition, we introduce the following functions on task queues:

- Sch is a sorting function for task queues (or lists), that may change the ordering of the queue elements only. For example, $\operatorname{EDF}([P(3.1,10), Q(4,5.3)])=$ $[Q(4,5.3), P(3.1,10)])$. We call such sorting functions a scheduling strategy that may be preemptive or non-preemptive 3 .

- Run is a function which given a real number $t$ and a task queue $q$ returns the resulted queue after $t$ time units of execution according to available resources. For simplicity, we assume that only one processor is available 4 . Then the meaning of $\operatorname{Run}(q, t)$ should be obvious and it can be defined inductively as follows: $\operatorname{Run}(q, 0)=q, \operatorname{Run}\left(\left[P_{0}\left(c_{0}, d_{0}\right), P_{1}\left(c_{1}, d_{1}\right) \ldots P_{n}\left(c_{n}, d_{n}\right)\right], t\right)$ $=\operatorname{Run}\left(\left[P_{1}\left(c_{1}, d_{1}-c_{0}\right) \ldots P_{n}\left(c_{n}, d_{n}-c_{0}\right)\right], t-c_{0}\right)$ if $c_{0} \leq t$ and $\operatorname{Run}\left(\left[P_{1}\left(c_{0}, d_{0}\right)\right.\right.$ $\left.\left.\ldots P_{n}\left(c_{n}, d_{n}\right)\right], t\right)=\left[P_{1}\left(c_{0}-t, d_{0}-t\right) \ldots P_{n}\left(c_{n}, d_{n}-t\right)\right]$ if $c_{0}>t$. For example, let $q=[Q(4,5), P(3,10)]$. Then $\operatorname{Run}(q, 6)=[P(1,4)]$ in which the first task is finished and the second has been executed for 2 time units.

We use $u=g$ to denote that the clock assignment $u$ satisfies the constraint $g$. For $t \in \mathcal{R}_{\geq 0}$, we use $u+t$ to denote the clock assignment which maps each clock $x$ to the value $u(x)+t$, and $u[r \mapsto 0]$ for $r \subseteq \mathcal{C}$, to denote the clock assignment which maps each clock in $r$ to 0 and agrees with $u$ for the other clocks (i.e. $\mathcal{C} \backslash r)$. Now we are ready to present the operational semantics for extended timed automata by transition rules:

Definition 2. Given a scheduling strategy Sch, the semantics of an automaton $\left\langle N, l_{0}, E, I, M\right\rangle$ with initial state $\left(l_{0}, u_{0}, q_{0}\right)$ is a labelled transition system defined by the following rules:

$$
\begin{aligned}
& -(l, u, q) \stackrel{a}{\longrightarrow} \operatorname{Sch}(m, u[r \mapsto 0], \operatorname{Sch}(M(m):: q)) \text { if } l \stackrel{\text { gar }}{\longrightarrow} m \text { and } u=g \\
& -(l, u, q) \stackrel{t}{\longrightarrow} \operatorname{Sch}(l, u+t, \operatorname{Run}(q, t)) \text { if }(u+t) \models I(l)
\end{aligned}
$$

where $M(m):: q$ denotes the queue with $M(m)$ inserted in $q$.

Note that the transition rules are parameterized by Sch (scheduling strategy). and Run (function representing the available computing resources). According to the transition rules, the task queue is growing with action transitions and shrinking with delay transitions. Multiple copies (instances) of the same task type may appear in the queue.

\footnotetext{
${ }^{3}$ A non-preemptive strategy will never change the position of the first element of a queue and a preemtive strategy may change the ordering of task types only, but never change the ordering of task instances of the same type.

${ }^{4}$ The semantics may be extended to multi-processor setting by modifying the function Run according the number of processors available.
} 
Whenever it is understood from the context, we shall omit Sch from the transition relation. Consider the automaton in Figure 2(b). Assume that preemptive earliest deadline first (EDF) is used to schedule the task queue. Then the automaton with initial state $\left(m_{1},[x=0],\left[Q_{1}(1,2)\right]\right)$ may demonstrate the following sequence of typical transitions:

$$
\begin{aligned}
& \left(m_{1},[x=0],\left[Q_{1}(1,2)\right]\right) \\
& \stackrel{1}{\longrightarrow}\left(m_{1},[x=1],\left[Q_{1}(0,1)\right]\right)=\left(m_{1},[x=1],[]\right) \\
& \stackrel{9.5}{\longrightarrow}\left(m_{1},[x=10.5],[]\right) \\
& \stackrel{a_{1}}{\longrightarrow}\left(n_{1},[x=0],\left[P_{1}(4,20)\right]\right) \\
& \stackrel{0.5}{\longrightarrow}\left(n_{1},[x=0.5],\left[P_{1}(3.5,19.5)\right]\right) \\
& \stackrel{b 2}{\longrightarrow}\left(m_{2},[x=0.5],\left[Q_{2}(1,4), P_{1}(3.5,19.5)\right]\right) \\
& \stackrel{0.3}{\longrightarrow}\left(m_{2},[x=0.8],\left[Q_{2}(0.7,3.7), P_{1}(3.5,19.2)\right]\right) \\
& \stackrel{a_{2}}{\longrightarrow}\left(n_{2},[x=0],\left[Q_{2}(0.7,3.7), P_{2}(2,10), P_{1}(3.5,19.2)\right]\right) \\
& \stackrel{b_{1}}{\longrightarrow}\left(m_{1},[x=0],\left[Q_{2}(0.7,3.7), Q_{1}(1,2), P_{2}(2,10), P_{1}(3.5,19.2)\right]\right) \\
& \stackrel{10}{\longrightarrow}\left(n_{1},[x=10],[]\right) \\
& \text {... }
\end{aligned}
$$

This is only a partial behaviour of the automaton. A question of interest is whether it can perform a sequence of transitions leading to a state where the task queue is non-schedulable.

\section{Schedulability Analysis}

In this section we study verification problems related to the model presented in the previous section. First, we have the same notion of reachability as for timed automata.

Definition 3. We shall write $(l, u, q) \longrightarrow\left(l^{\prime}, u^{\prime}, q^{\prime}\right)$ if $(l, u, q) \stackrel{a}{\longrightarrow}\left(l^{\prime}, u^{\prime}, q^{\prime}\right)$ for an action a or $(l, u, q) \stackrel{t}{\longrightarrow}\left(l^{\prime}, u^{\prime}, q^{\prime}\right)$ for a delay $t$. For an automaton with initial state $\left(l_{0}, u_{0}, q_{0}\right),(l, u, q)$ is reachable iff $\left(l_{0}, u_{0}, q_{0}\right) \longrightarrow *(l, u, q)$.

In general, the task queue is unbounded though the constraints of a givn automaton may restrict the possibility of reaching states with infinitely many different task queues. This makes the analysis of automata more difficult. However, for certain analysis, e.g. verification of safety properties that are not related to the task queue, we may only be interested in the reachability of locations. A nice property of our extension is that the location reachability problem can be checked by the same technique as for timed automata 1419 . So we may view the original timed automaton (without task assignment) as an abstraction of its extended version preserving location reachability. The existing model checking tools such as 2017] can be applied directly to verify the abstract models.

But if properties related to the task queue are of interests, we need to develop new verification techniques. One of the most interesting properties of extended automata related to the task queue is schedulability. 
Definition 4. (Schedulability) A state $(l, u, q)$ with $q=\left[P_{1}\left(c_{1}, d_{1}\right) \ldots\right.$ $\left.P_{n}\left(c_{n}, d_{n}\right)\right]$ is a failure denoted $\left(l, u\right.$, Error) if there exists $i$ such that $c_{i} \geq$ 0 and $d_{i}<0$, that is, a task failed in meeting its deadline. Naturally an automaton $A$ with initial state $\left(l_{0}, u_{0}, q_{0}\right)$ is non-schedulable with Sch iff $\left(l_{0}, u_{0}, q_{0}\right)(\longrightarrow \mathrm{Sch})^{*}(l, u$, Error $)$ for some $l$ and $u$. Otherwise, we say that $A$ is schedulable with Sch. More generally, we say that $A$ is schedulable iff there exists a scheduling strategy Sch with which $A$ is schedulable.

The schedulability of a state may be checked by the standard test. We say that $(l, u, q)$ is schedulable with $\operatorname{Sch}$ if $\operatorname{Sch}(q)=\left[P_{1}\left(c_{1}, d_{1}\right) \ldots P_{n}\left(c_{n}, d_{n}\right)\right]$ and $\left(\sum_{i<k} c_{i}\right) \leq d_{k}$ for all $k \leq n$. Alternatively, an automaton is schedulable with Sch if all its reachable states are schedulable with Sch.

Checking schedulability of a state is a trivial task according to the definition. But checking the relative schedulability of an automaton with respects to a given scheduling strategy is not easy, and checking the general schedulability (equivalent to finding a scheduling strategy to schedule the automaton) is even more difficult.

Fortunately the queues of all schedulable states of an automaton are bounded. First note that a task instance that has been started can not be preempted by another instance of the same task type. This means that there is only one instance of each task type in the queue whose computing time can be a real number and it can be arbitrarily small. Thus the number of instances of each task type $P \in \mathcal{P}$, in a schedulable queue is bounded by $\lceil D(P) / C(P)\rceil$ and the size of schedulable queues is bounded by $\sum_{P \in \mathcal{P}}\lceil D(P) / C(P)\rceil$.

We will code schedulability checking problems as reachability problems. First, we consider the case of non-preemptive scheduling to introduce the problems. We have the following positive result.

Theorem 1. The problem of checking schedulability relative to non-preemptive scheduling strategy for extended timed automata is decidable.

Proof. A detailed proof is given in 11. We sketch the proof idea here. It is to code the given scheduling strategy as a timed automaton (called the scheduler) denoted $E(\mathrm{Sch})$ which uses clocks to remember computing times and relative deadlines for released tasks. The scheduler automaton is constructed as follows: Whenever a task instance $P_{i}$ is released by an event release ${ }_{i}$, a clock $d_{i}$ is reset to 0 . Whenever a task is started to run, a clock $c$ is reset to 0 . Whenever the constraint $d_{i}=0$ is satisfied, and $P_{i}$ is not running, an error-state (non-schedulable) should be reached. We also need to transform the original automaton $A$ to $E(A)$ to synchronize with the scheduler that $P_{i}$ is released whenever a location, say $l$ to which $P_{i}$ is associated, is reached. This is done simply by replacing actions labeled on transitions leading to $l$ with release ${ }_{i}$. Finally we construct the product automaton $E(\mathrm{Sch}) \| E(A)$ in which both $E(\mathrm{Sch})$ and $E(A)$ can only synchronize on identical action symbols namely release ${ }_{i}$ 's. It can be proved that if an error-state of the product automaton is reachable, the original extended timed automaton is non-schedulable.

For preemptive scheduling strategies, it has been conjectured that the schedulability checking problem is undecidable. The reason is that if we use the same 
ideas as for non-preemptive scheduling to encode a preemptive scheduling strategy, we must use stop-watches (or integrators) to add up computing times for suspended tasks. It appears that the computation model behind preemptive scheduling is stop-watch automata for which it is known that the reachability problem is undecidable. Surprisingly this conjecture is wrong.

Theorem 2. The problem of checking schedulability relative to a preemptive scheduling strategy for extended timed automata is decidable.

The rest of this paper will be devoted to the proof of this theorem. It follows from Lemma 3, 4, and 5] established in the following section. Before we go further, we state a more general result that follows from the above theorem.

Theorem 3. The problem of checking schedulability for extended timed automata is decidable.

From scheduling theory [8], we know that the preemptive version of Earliest Deadline First scheduling (EDF) is optimal in the sense that if a task queue is non-schedulable with EDF, it can not be schedulable with any other scheduling strategy (preemptive or non-preemptive). Thus, the general schedulability checking problem is equivalent to the relative schedulability checking with respects to EDF.

\section{Decidability and Proofs}

We shall encode the schedulability checking problem as a reachability problem. For the case of non-preemptive scheduling, the expressive power of timed automata is enough. For preemptive scheduling, we need a more expressive model.

\subsection{Timed Automata with Subtraction}

Definition 5. A timed automaton with subtraction is a timed automaton in which clocks may be updated by subtraction in the form $x:=x-C$ in addition to reset of the form $x:=0$, where $C$ is a natural number.

This is the so called updatable automata 7]. It is known that the reachability problem for this class of automata is undecidable. However, for the following class of suspension automata, location reachability is decidable.

Definition 6. (Bounded Timed Automata with Subtraction) A timed automaton is bounded iff for all its reachable states $(l, u, q)$, there is a maximal constant $C_{x}$ for each clock $x$ such that

1. $u(x) \geq 0$ for all clocks $x$, i.e. clock values should not be negative and

2. $u(x) \leq C_{x}$ if $l \stackrel{\text { gar }}{\longrightarrow} l^{\prime}$ for some $l^{\prime}$ and $C$ such that $g(u)$ and $(x:=x-C) \in r$. 
In general, it may be difficult to compute the maximal constants from the syntax of an automaton. But we shall see that we can compute the constants for our encoding of scheduling problems.

Because subtractions on clocks are performed only within a bounded area, the region equivalence is preserved by the operation. We adopt the standard definition due to Alur and Dill [5].

Definition 7. (Region Equivalence denoted $\sim$ ) For a clock $x \in \mathcal{C}$, let $C_{x}$ be a constant (the ceiling of clock $x$ ). For a real number $t$, let $\{t\}$ denote the fractional part of $t$, and $\lfloor t\rfloor$ denote its integer part. For clock assignments $u, v \in \mathcal{V}, u, v$ are region-equivalent denote $u \sim v$ iff

1. for each clock $x$, either $\lfloor u(x)\rfloor=\lfloor v(x)\rfloor$ or $u(x)>C_{x}$ and $\left.v(x)>C_{x}\right)$, and

2. for all clocks $x, y$ if $u(x) \leq C_{x}$ and $u(y) \leq C_{y}$ then

a) $(\{u(x)\}=0$ iff $\{v(x)\}=0$ and

b) $\{u(x)\} \leq\{u(y)\}$ iff $\{v(x)\} \leq\{v(y)\}$

It is known that region equivalence is preserved by the delay (addition) and reset. In the following, we establish that region equivalence is also preserved by subtraction for clocks that are bounded as defined in Definition 6. For a clock assignment $u$, let $u(x-C)$ denote the assignment: $u(x-C)(x)=u(x)-C$ and $u(x-C)(y)=u(y)$ for $y \neq x$.

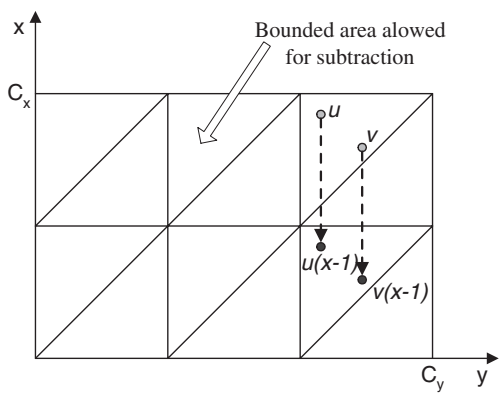

Fig. 3. Region equivalence preserved by subtraction when clocks are bounded.

Lemma 1. Let $u, v \in \mathcal{V}$. Then $u \sim v$ implies

1. $u+t \sim v+t$ for a positive real number $t$, and

2. $u[x \mapsto 0] \sim v[x \mapsto 0]$ for a clock $x$ and

3. $u(x-C) \sim v(x-C)$ for all natural numbers $C$ such that $C \leq u(x) \leq C_{x}$.

Proof. It is given in the full version of this paper [13].

In fact, region equivalence over clock assignments induces a bisimulation over reachable states of automata, which can be used to partition the whole state space as a finite number of equivalence classes. 
Lemma 2. Assume a bounded timed automaton with subtraction, a location $l$ and clock assignments $u$ and $v$. Then $u \sim v$ implies that

1. whenever $(l, u) \longrightarrow\left(l^{\prime}, u^{\prime}\right)$ then $(l, v) \longrightarrow\left(l^{\prime}, v^{\prime}\right)$ for some $v^{\prime}$ s.t. $u^{\prime} \sim v^{\prime}$ and

2. whenever $(l, v) \longrightarrow\left(l^{\prime}, v^{\prime}\right)$ then $(l, u) \longrightarrow\left(l^{\prime}, u^{\prime}\right)$ for some $u^{\prime}$ s.t. $u^{\prime} \sim v^{\prime}$.

Proof. It follows from Lemma 1

The above lemma essentially states that if $u \sim v$ then $(l, u)$ and $(l, v)$ are bisimular, which implies the following result.

Lemma 3. The location reachability problem for bounded timed automata with subtraction, whose clocks are bounded with known maximal constants is decidable.

Proof. Because each clock of the automaton is bounded by a maximal constant, it follows from lemma 2 that for each location $l$, there is a finite number of equivalence classes of states which are equivalent in the sense that they will reach the same equivalence classes of states. Because the number of locations of an automaton is finite, the whole state space of an automaton can be partitioned into finite number of such equivalence classes.

\subsection{Encoding of Schedulability as Reachability}

Assume an automaton $A$ extended with tasks, and a preemptive scheduling strategy Sch. The aim is to check if $A$ is schedulable with Sch. As for the case of non-preemptive scheduling (Theorem 11), we construct $E(A)$ and $E(\mathrm{Sch})$, and check a pre-defined error-state in the product automaton of the two. The construction is illustrated in figure 4 .

$E(A)$ is constructed as a timed automaton which is exactly the same as for the non-preemptive case (Theorem 1) and $E(\mathrm{Sch})$ will be constructed as a timed automaton with subtraction.

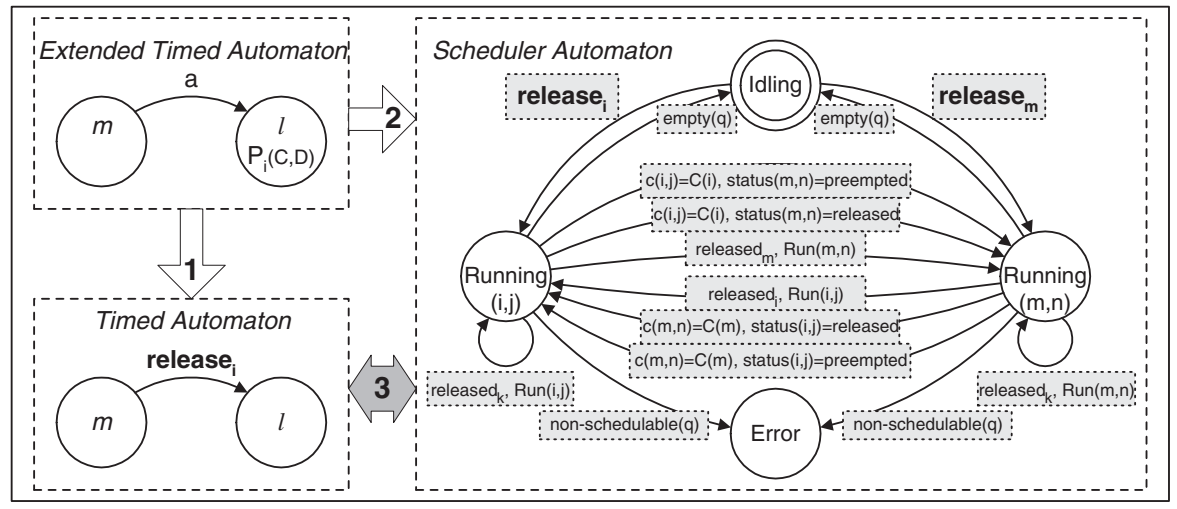

Fig. 4. Encoding of schelulability problem. 
We introduce some notation. Let $C(i)$ and $D(i)$ stand for the worst case execution time and relative deadline respectively for each task type $P_{i}$. We use $P_{i j}$ to denote the $j$ th instance of task type $P_{i}$.

For each task instance $P_{i j}$, we have the following state variables: status $(i, j)$ initialized to free. Let status $(i, j)=$ running stand for that $P_{i j}$ is executing on the processor, preempted for that $P_{i j}$ is started but not running, and released for that $P_{i j}$ is released, but not started yet. We use status $(i, j)=$ free to denote that $P_{i j}$ is not released yet or position $(i, j)$ of the task queue is free.

According to the definition of scheduling strategy, for all $i$, there should be only one $j$ such that status $(i, j)=$ preempted (only one instance of the same task type is started), and for all $i, j$, there should be only one pair $(k, l)$ such that $\operatorname{status}(k, l)=$ running (only one is running for a one-processor system).

We need two clocks for each task instance:

1. $c(i, j)$ (a computing clock) is used to remember the accumulated computing time since $P_{i j}$ was started (when $\operatorname{Run}(i, j)$ became true), and subtracted with $C(k)$ when the running task, say $P_{k l}$, is finished if it was preempted after it was started.

2. $d(i, j)$ (a deadline clock) is used to remember the deadline and reset to 0 when $P_{i j}$ is released.

We use a triple $\langle c(i, j), d(i, j)$, status $(i, j)\rangle$ to represent each task instance, and the task queue will contain such triples. We use $q$ to denote the task queue. Note that the maximal number of instances of $P_{i}$ appearing in a schedulable queue is $\lceil D(i) / C(i)\rceil$. We have a bound on the size of queue as claimed earlier, which is $\sum_{P_{i} \in \mathcal{P}}\lceil D(i) / C(i)\rceil$. We shall say that queue is empty denoted empty $(q)$ if $\operatorname{status}(i, j)=$ free for all $i, j$.

For a given scheduling strategy Sch, we use the predicate $\operatorname{Run}(m, n)$ to denote that task instance $P_{m n}$ is scheduled to run according to Sch. For a given Sch, it can be coded as a constraint over the state variables. For example, for EDF, $\operatorname{Run}(m, n)$ is the conjunction of the following constraints:

1. $d(k, l) \leq D(k)$ for all $k, l$ such that $\operatorname{status}(k, l) \neq$ free: no deadline is violated yet

2. $\operatorname{status}(m, n) \neq$ free: $P_{m n}$ is released or preempted

3. $D(m)-d(m, n) \leq D(i)-d(i, j)$ for all $(i, j): P_{m n}$ has the shortest deadline

$E$ (Sch) contains three type of locations: Idling, Running and Error with Running being parameterized with $(i, j)$ representing the running task instance.

1. Idling denotes that the task queue is empty.

2. Running $(i, j)$ denotes that task instance $P_{i j}$ is running, that is, $\operatorname{status}(i, j)=$ running. We have an invariant for each Running $(i, j): c(i, j) \leq C(i)$ and $d(i, j) \leq D(i)$.

3. Error denotes that the task queues are non-schedulable with Sch.

There are five types of edges labeled as follows:

\footnotetext{
${ }^{5}$ In fact, for each task type, we need only one clock for computing time because only one instance of the same task type may be started.
} 
1. Idling to Running $(i, j)$ : there is an edge labeled by

- guard: none

- action: release ${ }_{i}$

- reset: $c(i, j):=0, d(i, j):=0$, and $\operatorname{status}(i, j):=$ running

2. Running $(i, j)$ to Idling: there is only one edge labeled with

- guard: $\operatorname{empty}(q)$ that is, $\operatorname{status}(i, j)=$ free for all $i, j$ (all positions are free).

- action: none

- reset: none

3. Running $(i, j)$ to $\operatorname{Running}(m, n)$ : there are two types of edges.

a) The running task $P_{i j}$ is finished, and $P_{m n}$ is scheduled to run by $\operatorname{Run}(m, n)$. There are two cases:

i. $P_{m n}$ was preempted earlier:

- guard: $c(i, j)=C(i), \operatorname{status}(m, n)=\operatorname{preempted}$ and $\operatorname{Run}(m, n)$

- action: none

- reset: $\operatorname{status}(i, j):=$ free, $\{c(k, l):=c(k, l)-C(i) \mid \operatorname{status}(k, l)=$ preempted $\}$, and $\operatorname{status}(m, n):=$ running

ii. $P_{m n}$ was released, but never preempted (not started yet):

- guard: $c(i, j)=C(i)$, status $(m, n)=$ released and $\operatorname{Run}(m, n)$

- action: none

- reset: $\operatorname{status}(i, j):=$ free, $\{c(k, l):=c(k, l)-C(i) \mid \operatorname{status}(k, l)=$ preempted $\}, c(m, n):=0, d(m, n):=0$ and $\operatorname{status}(m, n):=$ running

b) A new task $P_{m n}$ is released, which preempts the running task $P_{i j}$ :

- guard: $\operatorname{status}(m, n)=$ free, and $\operatorname{Run}(m, n)$

- action: released ${ }_{m}$

- reset: $\operatorname{status}(m, n):=$ running, $c(m, n):=0, d(m, n):=0$, and $\operatorname{status}(i, j):=$ preempted

4. Running $(i, j)$ to $\operatorname{Running}(i, j)$. There is only one edge representing the case when a new task is released, and the running task $P_{i j}$ will continue to run:

- guard: $\operatorname{status}(k, l)=$ free, and $\operatorname{Run}(i, j)$

- action: released $k$

- reset: $\operatorname{status}(k, l):=$ released and $d(k, l):=0$

5. Running $(i, j)$ to Error: for each pair $(k, l)$, there is an edge labeled by $d(k, l)>$ $D(k)$ and status $(k, l) \neq$ free meaning that the task $P_{k l}$ which is released (or preempted) fails in meeting its deadline.

The third step of the encoding is to construct the product automaton $E(S c h) \| E(A)$ in which both $E(S c h)$ and $E(A)$ can only synchronize on identical action symbols. Now we show that the product automaton is bounded.

Lemma 4. All clocks of $E(\mathrm{Sch})$ in $E(S c h) \| E(A)$ are bounded and nonnegative.

Proof. It is given in the full version of this paper [13].

Now we have the correctness lemma for our encoding. Assume, without losing generality, that the initial task queue of an automaton is empty. 
Lemma 5. Let $A$ be an extended timed automaton and Sch a scheduling strategy. Assume that $\left(l_{0}, u_{0}, q_{0}\right)$ and $\left(\left\langle l_{0}, \mathrm{Idling}\right\rangle, u_{0}\right)$ are the initial states of $A$ and the product automaton $E(A) \| E(\mathrm{Sch})$ respectively where $l_{0}$ is the initial location of $A, u_{0}$ and $v_{0}$ are clock assignments assigning all clocks with 0 and $q_{0}$ is the empty task queue. Then for all $l$ and $u$ :

$\left(l_{0}, u_{0}, q_{0}\right) \longrightarrow{ }^{*}(l, u$, Error $)$ iff $\left(\left\langle l_{0}\right.\right.$, Idling $\left.\rangle, u_{0} \cup v_{0}\right) \longrightarrow^{*}(\langle l$, Error $\rangle, u \cup v)$ for some $v$

Proof. It is by induction on the length of transition sequence.

The above lemma states that the schedulability analysis problem can be solved by reachability analysis for timed automata extended with subtraction. From Lemma 4 we know that $E(\mathrm{Sch})$ is bounded. Because the reachability problem is decidable due to Lemma 3, we complete the proof for our main result stated in Theorem 2,

\section{Conclusions and Related Work}

We have studied a model of timed systems, which unifies timed automata with the classic task models from scheduling theory. The model can be used to specify resource requirements and hard time constraints on computations, in addition to features offered by timed automata. It is general and expressive enough to describe concurrency and synchronization, and tasks which may be periodic, sporadic, preemptive and (or) non-preemptive. The classic notion of schedulability is naturally extended to automata model.

Our main technical contribution is the proof that the schedulability checking problem is decidable. The problem has been suspected to be undecidable due to the nature of preemptive scheduling. To our knowledge, this is the first decidability result for preemptive scheduling in dense-time models. Based the proof, we have developed a symbolic schedulability checking algorithm using the DBM techniques extended with a subtraction operation. It has been implemented in a prototype tool 6]. We believe that our work is one step forward to bridge scheduling theory and automata-theoretic approaches to system modeling and analysis. A challenge is to make the results an applicable technique combined with classic methods such as rate monotonic scheduling. We need new algorithms and data structures to represent and manipulate the dynamic task queue consisting of time and resource constraints. As another direction of future work, we shall study the schedule synthesis problem. More precisely given an automaton, it is desirable to characterize the set of schedulable traces accepted by the automaton.

Related work. Scheduling is a well-established area. Various analysis methods have been published in the literature. For systems restricted to periodic tasks, algorithms such as rate monotonic scheduling are widely used and efficient methods for schedulability checking exist, see e.g. [8. These techniques can be used to handle non-periodic tasks. The standard way is to consider non-periodic tasks as periodic using the estimated minimal inter-arrival times as task periods. Clearly, 
the analysis based on such a task model would be pessimistic in many cases, e.g. a task set which is schedulable may be considered as non-schedulable as the interarrival times of the tasks may vary over time, that are not necessary minimal. Our work is more related to work on timed systems and scheduling.

A nice work on relating classic scheduling theory to timed systems is the controller synthesis approach 23. The idea is to achieve schedulability by construction. A general framework to characterize scheduling contstraints as invariants and synthesize scheduled systems by decomposition of constraints is presented in [3]. However, algorithmic aspects are not discussed in these work. Timed automata has been used to solve non-preemptive scheduling problems mainly for job-shop scheduling $1,12,15]$. These techniques specify pre-defined locations of an automaton as goals to achieve by scheduling and use reachability analysis to construct traces leading to the goal locations. The traces are used as schedules. There have been several work e.g. [18,109] on using stop-watch automata to model preemptive scheduling problems. As the reachability analysis problem for stop-watch automata is undecidable in general 4], there is no guarantee for termination for the analysis without the assumption that task preemptions occur only at integer points. The idea of subtractions on timers with integers, was first proposed by McManis and Varaiya in [18. In general, the class of timed automata with subtractions is undecidable, which is shown in [7]. In this paper, we have identified a decidable class of updatable automata, which is precisely what we need to solve scheduling problems without assuming that preemptions occur only at integer points.

Acknowledgement. Thanks to the anonymous referees for their insights and constructive comments.

\section{References}

1. Y. Abdeddaïm and O. Maler. Job-shop scheduling using timed automata. In Proceedings of 13th Conference on Computer Aided Verification, July 18-23, 2001 Paris, France, 2001.

2. K. Altisen, G. Gößler, A. Pnueli, J. Sifakis, S. Tripakis, and S. Yovine. A framework for scheduler synthesis. In Proceedings of the 20th IEEE Real-Time Systems Symposium, Phoenix, AZ, USA, 1-3 December, 1999, pages 154-163. IEEE Computer Society Press, 1999.

3. K. Altisen, G. Gößler, and J. Sifakis. A methodology for the construction of scheduled systems. In Proceedings of FTRTFT 2000, Pune, India, September 2000, LNCS 1926, pp.106-120, 2000.

4. R. Alur, C. Courcoubetis, N. Halbwachs, T. A. Henzinger, P.-H. Ho, X. Nicollin, A. Olivero, J. Sifakis, and S. Yovine. The algorithmic analysis of hybrid systems. Theoretical Computer Science, 138(1):3-34, 1995.

5. R. Alur and D. L. Dill. A theory of timed automata. Theoretical Computer Science, 126(2):183-235, 1994.

6. T. Amnell, E. Fersman, L. Mokrushin, P. Pettersson, and W. Yi. TIMES - a tool for modelling and implementation of embedded systems. In Proceedings of TACAS02. Springer-Verlag, 2002. 
7. P. Bouyer, C. Dufourd, E. Fleury, and A. Petit. Are timed automata updatable? In Proceedings of the 12th International Conference on Computer-Aided Verification, Chicago, IL, USA, July 15-19, 2000, 2000. Springer-Verlag.

8. G. C. Buttazzo. Hard Real-Time Computing Systems. Predictable Scheduling Algorithms and Applications. Kulwer Academic Publishers, 1997.

9. F. Cassez and F. Laroussinie. Model-checking for hybrid systems by quotienting and constraints solving. In Proceedings of the 12th International Conference on Computer-Aided Verification, pages 373-388, Standford, California, USA, 2000. Springer-Verlag.

10. J. Corbett. Modeling and analysis of real-time ada tasking programs. In Proceedings of 15th IEEE Real-Time Systems Symposium, San Juan, Puerto Rico, USA, pages 132-141. IEEE Computer Society Press, 1994.

11. C. Ericsson, A. Wall, and W. Yi. Timed automata as task models for eventdriven systems. In Proceedings of the 6th International Conference on Real-Time Computing Systems and Applications (RTCSA'99). IEEE Computer Society Press, 1999.

12. A. Fehnker. Scheduling a steel plant with timed automata. In Proceedings of the 6th International Conference on Real-Time Computing Systems and Applications (RTCSA'99). IEEE Computer Society Press, 1999.

13. E. Fersman, P. Pettersson, and W. Yi. Timed automata with asynchronous processes: Schedulability and decidability. Technical report, Department of Information Technology, Uppsala University, Sweden, 2002.

14. T. Henzinger, X. Nicollin, J. Sifakis, and S. Yovine. Symbolic model checking for real-time systems. Information and Computation, 111(2):193-244, 1994.

15. T. Hune, K. G. Larsen, and P. Pettersson. Guided Synthesis of Control Programs using UpPaAl. Nordic Journal of Computing, 8(1):43-64, 2001.

16. K. G. Larsen, P. P., and W. Yi. Compositional and symbolic model-checking of real-time systems. In Proceedings of 16th IEEE Real-Time Systems Symposium, December 5-7, 1995 - Pisa, Italy, pages 76-89. IEEE Computer Society Press, 1995.

17. K. G. Larsen, P. Pettersson, and W. Yi. UppaAL in a Nutshell. Journal on Software Tools for Technology Transfer, 1(1-2):134-152, October 1997.

18. J. McManis and P. Varaiya. Suspension automata: a decidable class of hybrid automata. In Proceedings of the 6th International Conference on Computer-Aided Verification, pages 105-117, Standford, California, USA, 1994. Springer-Verlag.

19. W. Yi, P. Pettersson, and M. Daniels. Automatic verification of real-time communicating systems by constraint-solving. In Proceedings of the 7th International Conference on Formal Description Techniques, 1994.

20. Sergio Yovine. A Verification Tool for Real Time Systems. Int. Journal on Software Tools for Technology Transfer, 1(1-2):134-152, October 1997. 